\title{
Confiabilidade e validade concorrente da Escala de Mobilidade Hospitalar em pacientes após acidente vascular cerebral
}

\author{
Reliability and concurrent validity of the Hospital \\ Mobility Scale in acute stroke patients
}

\author{
Iara Maso' 1 (i) \\ Laísa Mascarenhas ${ }^{2}$ (]) \\ Marina Makhoul 3 (1) \\ Lara Vasconcelos ${ }^{4}$ \\ Maiana Monteiro 5 (1) \\ Pedro Jesus $^{6}$ (b) \\ Jamary Oliveira-Filho ${ }^{7}$ \\ Elen Beatriz Pinto ${ }^{8}$ (1)
}

\begin{abstract}
'Autora para correspondência. Escola Bahiana de Medicina e Saúde Pública (Salvador), Hospital Geral Roberto Santos (Salvador). Bahia, Brasil. iaramaso@gmail.com

2-5,8Escola Bahiana de Medicina e Saúde Pública (Salvador). Bahia, Brasil. laisasm.fisio@gmail.com,mp_makhoul@hotmail.com, lara_vasconceloss@hotmail.com, maidellacella@yahoo.com.br, elen.neuro@gmail.com ${ }^{6}$ Hospital Geral Roberto Santos (Salvador). Bahia, Brasil. papj@terra.com.br ־Universidade Federal da Bahia (Salvador). Bahia, Brasil. jamary@mail.harvard.edu
\end{abstract}

RESUMO | INTRODUÇÃO: A Escala de Mobilidade Hospitalar (EMH) avalia de forma específica a mobilidade de pacientes após AVC no ambiente hospitalar e em estudo prévio foi demonstrada a sua concordância interexaminadores, validade preditiva e a responsividade a mudanças na fase aguda. OBJETIVO: Avaliar a validade concorrente da EMH e a sua confiabilidade ao ser aplicada através de entrevista. MATERIAIS E MÉTODOS: Trata-se de um estudo de validação, desenvolvido com pacientes internados em uma Unidade de AVC na cidade de Salvador-Bahia. Para avaliar a validade concorrente da EMH comparamos o seu escore com a pontuação da Escala de Rankin modificada (ERm) utilizando o teste de Spearman. Ambas escalas foram aplicadas no quinto dia após o AVC pelo mesmo pesquisador, previamente treinado. Para avaliação da confiabilidade da EMH quando aplicada através de entrevista, a escala foi aplicada por dois examinadores em turnos diferentes, no mesmo dia. O primeiro examinador aplicou a escala através da observação do desempenho e o segundo examinador através de entrevista. Utilizamos para esta análise o Coeficiente de Correlação Intraclasse (CCI). RESULTADOS: A pontuação total da EMH apresentou uma correlação positiva muito forte com a $\operatorname{ERm}(r=0,90)$ e também foi encontrada uma correlação significativa entre os subitens da $E M H$ e a ERm. Ao comparar a aplicação da EMH através da observação do desempenho e aplicação por entrevista, observamos uma excelente concordância interexaminadores $(\mathrm{CCl}>0,90)$. CONCLUSÃo: A Escala de Mobilidade Hospitalar, projetada especificamente para pacientes após AVC, mostrou um alto grau de validade concorrente e se mostrou confiável quando aplicada através de entrevista.

PALAVRAS-CHAVE: Acidente vascular cerebral. Mobilização precoce. Confiabilidade. Estudos de validação.
ABSTRACT | INTRODUCTION: The Hospital Mobility Scale (HMS) evaluates the mobility of stroke patients in the hospital environment and in a previous study showed its inter-examiner agreement, predictive validity and responsiveness to changes in the acute phase. OBJECTIVE: To evaluate the concurrent validity of HMS and its reliability when applied by interview. METHODS: This is a validation study, that was developed with patients admitted in a stroke unit, in the city of Salvador, Bahia, Brazil. In order to assess the concurrent validity of the HMS, we compared its score with the modified Rankin Scale (mRS) score by using the spearman test. The same researcher applied both scales on the fifth day after stroke. To assess the reliability of HMS when applied by interview, two examiners applied the scale in different shifts, on the same day. The first evaluation was face-to-face and the second was performed by interview. For this analysis, we used the intraclass correlation coefficient (ICC). RESULTS: The HMS showed a very strong positive correlation with the mRS ( $r=0.90)$ and a significant correlation was also found between the sub-items of the HMS and the mRS. We found excellent inter-examiner agreement between face-to-face and interview assessment (ICC> 0.90). CONCLUSION: The hospital mobility scale, that was developed specifically for stroke patients, showed a high degree of concurrent validity and was reliable when applied by interview.

KEYWORDS: Stroke. Early mobilization. Reliability. Validation studies. 


\section{Introdução}

A avaliação da mobilidade em pacientes hospitalizados é essencial para definição de condutas fisioterapêuticas e acompanhamento da evolução dos pacientes, especialmente em indivíduos após acidente vascular cerebral (AVC), que podem apresentar limitações na mobilidade durante a fase aguda ${ }^{1}$. Neste cenário, encontram-se algumas escalas de mobilidade validadas para população após AVC ${ }^{2,3}$. Entretanto, estes instrumentos exigem na sua aplicação um ambiente padronizado e equipamentos ${ }^{2}$, que mesmo parecendo simples e acessíveis, podem não estar disponíveis na rotina dos hospitais, especialmente em serviços com poucos recursos.

Nos últimos anos surgiram algumas escalas de mobilidades desenvolvidas para avaliação de pacientes internados em unidades de terapia intensiva 4,5 . Estas escalas são unidimensionais e avaliam a mobilidade como uma tarefa única que varia desde a restrição ao leito (pontuação zero) até a marcha (pontuações máximas $)^{4,5}$. Essa graduação do nível de mobilidade se adequa bem ao ambiente de terapia intensiva, mas é insuficiente para a avalição do paciente após AVC, uma vez que o controle postural em cada uma das posturas é bastante variável nestes pacientes.

Com intuito de propor um novo instrumento que avalia de forma específica a mobilidade de pacientes após AVC no ambiente hospitalar, a Escala de Mobilidade Hospitalar (EMH) foi desenvolvida e a sua validação publicada ${ }^{1}$. Este é um instrumento de rápida aplicação que avalia a mobilidade através de 3 tarefas (sedestação, ortostase e marcha), não requer treinamento específico, não necessita de equipamentos ou ambiente padronizado. Sendo assim, pode ser incluída facilmente na prática clínica para registro diário do nível de mobilidade, permitindo que o fisioterapeuta direcione $o$ atendimento para o mais alto nível de mobilidade. Além disso, diferente das demais escalas de mobilidade aqui referidas, a EMH inclui a possibilidade de contar com o auxílio de uma ou duas pessoas durante a sedestação, ortostase e marcha, o que representa uma realidade dos pacientes após AVC, e facilita o planejamento dos atendimentos nas unidades hospitalares ${ }^{1}$.
O estudo de Maso e colaboradores em 2019, mostrou a habilidade da EMH em prever o desfecho funcional de pacientes após 3 meses do ictus, além de demonstrar sua concordância interexaminadores e responsividade a mudanças na fase aguda. O objetivo do presente estudo é ampliar a avaliação das propriedades psicométricas da EMH investigando: 1. A validade concorrente, através da comparação da pontuação da EMH com a Escala de Rankin modificada; 2 . A confiabilidade da EMH quando aplicada através de entrevista.

\section{Materiais e métodos}

Trata-se de um estudo de validação, envolvendo pacientes internados na Unidade de AVC (UAVC) do Hospital Geral Roberto Santos, na cidade de SalvadorBahia. Esta unidade oferece atendimento multidisciplinar incluindo reabilitação para pacientes após AVC na fase aguda. Foram incluídos indivíduos com idade superior a 18 anos, com diagnóstico de AVC isquêmico e hemorrágico confirmado por neuroimagem (tomografia computadorizada ou ressonância nuclear magnética). Foram excluídos indivíduos com alterações funcionais prévias ao AVC que apresentavam pontuação < 95 no Índice de Barthel modificado, bem como pacientes previamente cegos, amputados, com demência ou outra patologia neurológica que comprometesse a mobilidade. Foi preenchido um questionário com dados coletados em prontuários, contendo variáveis clínicas e sociodemográficas.

Para avaliação da validade concorrente usamos o banco de dados de um estudo publicado previamente ${ }^{1}$ e para avaliação da confiabilidade utilizamos uma amostra de conveniência de paciente atendidos na UAVC no mesmo período do estudo citado.

\section{Avaliação da validade concorrente}

Para avaliar a validade concorrente da EMH comparamos o seu escore com a pontuação da Escala de Rankin modificada (ERm). Ambas escalas foram aplicadas no quinto dia após o AVC pelo mesmo pesquisador, que foi previamente treinado. 


\section{Avaliação da confiabilidade da EMH ao ser aplicada através de entrevista}

Para avaliação da confiabilidade, a EMH foi aplicada por dois examinadores em turnos diferentes, no mesmo dia. O primeiro examinador foi um fisioterapeuta que mobilizou o paciente durante seu atendimento e aplicou a escala observando o desempenho do paciente em cada tarefa. O segundo examinador aplicou a EMH ao final do plantão através de uma entrevista com o fisioterapeuta que fez o atendimento.

\section{Instrumentos de avaliação}

A Escala de Mobilidade Hospitalar (Figura 1) ${ }^{1}$ avalia o grau de dependência em três tarefas: sedestação, ortostase e marcha e cada tarefa é classificada de acordo com o grau de dependência (realiza de forma independente, necessita do auxílio de uma pessoa, necessita do auxílio de duas pessoas ou não consegue realizar a tarefa). Sua pontuação varia de 0 a 12, e quanto maior a pontuação, maior o grau de dependência ${ }^{1}$.

Figura 1. Escala de Mobilidade Hospitalar

\section{ESCALA DE MOBILIDADE HOSPITALAR (EMH)}

\begin{tabular}{|c|c|}
\hline Tarefas & Escore \\
\hline \multicolumn{2}{|l|}{ Sedestação no leito com membros inferiores pendentes } \\
\hline Consegue realizar a transferência e permanecer sentado de forma independente & 0 \\
\hline Necessita de auxílio de uma pessoa para permanecer sentado e/ou durante as transferências & 2 \\
\hline Necessita de auxílio de duas pessoas para permanecer sentado e/ ou durante as transferências & 4 \\
\hline Não consegue realizar a tarefa & 6 \\
\hline \multicolumn{2}{|l|}{ Ortostase } \\
\hline Consegue permanecer em ortostase encostado no leito sem auxílio & 0 \\
\hline Necessita de auxílio de uma pessoa para permanecer em ortostase encostado no leito e/ou durante as transferências & 1 \\
\hline Necessita de auxílio de duas pessoas para permanecer em ortostase encostado no leito e/ou durante as transferências & 2 \\
\hline Não consegue realizar a tarefa & 3 \\
\hline \multicolumn{2}{|l|}{ Marcha } \\
\hline Marcha independente & 0 \\
\hline Marcha com auxílio ou supervisão de uma pessoa & 1 \\
\hline Marcha com auxílio de duas pessoas & 2 \\
\hline Não consegue realizar a tarefa & 3 \\
\hline
\end{tabular}


A Escala de Rankin modificada descreve o grau de disfunção do paciente, incluindo a avaliação da marcha, atividades básicas e atividades habituais. Atribui uma pontuação que varia de 0 a 6, sendo 0 - Assintomático e 6 - Óbito ${ }^{6}$.

\section{Análise Estatística}

O programa SPSS versão 20.0 foi utilizado para estruturação da planilha de dados e para as análises estatísticas. Foi realizada uma análise descritiva das variáveis clínicas e demográficas com a finalidade de identificar as características da população estudada. As variáveis numéricas foram apresentadas em média e desvio padrão ou mediana e intervalo interquartil após verificação da normalidade através do teste de Kolmogorov-Smirnov. Utilizamos o teste de Spearman para avaliar a correlação entre os escores da EMH e ERm. Consideramos a seguinte classificação da intensidade de correlação: $r=0$ nula; $r \leq 0,30$ fraca; $0,30<r \leq 0,60$ regular; $0,60<r \leq 0,90$ forte; $0,90 \leq r<1$ muito forte; e $r=1$ plena ou perfeita ${ }^{7}$. Para comparar a pontuação da EMH aplicada através de observação de desempenho versus entrevista, utilizamos o Coeficiente de Correlação Intraclasse (CCI). O CCI varia de 0 a 1, e quanto maior o coeficiente, melhor a concordância. Consideramos como uma concordância pobre o CCl < 0,4; concordância satisfatória o 0,4 $\leq \mathrm{CCl}<0,75$; concordância excelente o 0,75 $\leq \mathrm{CCl}<1$; e concordância perfeita $\circ \mathrm{CCl}=1^{8}$. Valores de $\mathrm{p}<0,05$ foram considerados estatisticamente significativos.

\section{Aspectos Éticos}

Este estudo recebeu aprovação do conselho de revisão institucional local (protocolo CAAE: 27383014.9.0000.54) e todos os participantes incluídos forneceram um consentimento informado assinado.

\section{Resultados}

Para avaliação da validade concorrente foram avaliados 283 pacientes, com média de idade de 62,3 anos (+/-0,8) e $51,2 \%$ eram do sexo masculino (Tabela 1 ).

Tabela 1. Características demográficas e clínicas de 283 indivíduos admitidos em uma unidade de AVC, na cidade de Salvador, Bahia

\begin{tabular}{|c|c|}
\hline Variáveis & $N=283$ \\
\hline Idade em anos, média (DP) & $62,3( \pm 0,8)$ \\
\hline Sexo masculino, $\mathrm{n}(\%)$ & $145(51,2)$ \\
\hline Escolaridade em anos, mediana (IQ) & $6(4-12)$ \\
\hline Renda ( $n^{\circ}$ salários), mediana (IQ) & $2(1-2)$ \\
\hline AVC prévio, n (\%) & $70(24,7)$ \\
\hline \multicolumn{2}{|l|}{ Tipo } \\
\hline Isquêmico, n (\%) & $237(83,7)$ \\
\hline Isquêmico com transformação hemorrágica, n (\%) & $46(16,3)$ \\
\hline Tratamento trombolítico, $\mathrm{n}(\%)$ & $83(29,3)$ \\
\hline \multicolumn{2}{|l|}{ Local da lesão } \\
\hline Circulação anterior, $\mathrm{n}(\%)$ & $210(74,2)$ \\
\hline Circulação posterior, n (\%) & $43(15,2)$ \\
\hline Circulação anterior e posterior, n (\%) & $3(1,1)$ \\
\hline Sem lesão na tomografia após trombólise, n (\%) & $26(9,2)$ \\
\hline \multicolumn{2}{|l|}{ Gravidade do AVC (NIHSS) } \\
\hline Leve $(0-7), \mathrm{n}(\%)$ & $105(37,1)$ \\
\hline Moderada (8-16), n (\%) & $112(39,6)$ \\
\hline Grave $(>16), \mathrm{n}(\%)$ & $66(23,3)$ \\
\hline
\end{tabular}

Abreviações: SD, desvio padrão; IQ, interval interquartil; NIHSS, National Institutes of Health Stroke Scale. 
A pontuação total da EMH apresentou uma correlação positiva muito forte com a $\operatorname{ERm}(r=0,90)$, indicando que quanto maior o comprometimento da mobilidade maior o grau de incapacidade funcional. Uma correlação significativa também foi encontrada entre os subitens da EMH e a ERm (Tabela 2).

Tabela 2. Correlação entre o nível de mobilidade avaliado pela Escala de Mobilidade Hospitalar (EMH) e o grau de incapacidade avaliado pela Escala de Rankin modificada (ERm)

\begin{tabular}{lcc}
\hline & $\begin{array}{c}\text { Coeficiente de correlação de } \\
\text { Spearman }(r)\end{array}$ & $P$ \\
\hline Subitem sedestação da EMH x ERm & 0,79 & $<0,001$ \\
Subitem ortostase da EMH x ERm & 0,92 & $<0,001$ \\
Subitem marcha da EMH x ERm & 0,97 & $<0,001$ \\
Pontuação total da EMH x ERm & 0,90 & $<0,001$ \\
\hline
\end{tabular}

Para avaliar a confiabilidade da EMH quando aplicada através de entrevista, avaliamos uma amostra de 39 pacientes, com média de idade de 62,1 anos (+/-2,1), 56,4\% eram do sexo masculino, 81,6\% tiveram AVC isquêmico e $18,4 \%$ AVC hemorrágico.

Ao comparar a aplicação da EMH através da observação do desempenho e aplicação por entrevista, observamos uma excelente concordância interexaminadores $(\mathrm{CCl}>0,90)$ para a pontuação total, bem como para as tarefas sedestação e marcha. No subitem ortostase encontramos uma concordância perfeita $(\mathrm{CCl}=1)$, o que indica que os dois examinadores tiveram as mesmas respostas no item ortostase para todos os pacientes (Tabela 3).

Tabela 3. Confiabilidade da EMH quando aplicada através da observação de desempenho versus entrevista

\begin{tabular}{lccc}
\hline & $\mathrm{CCI}^{*}$ & Intervalo de confiança & $\mathrm{P}$ \\
\hline Sedestação & 0,96 & $(0,92-0,98)$ & $<0,001$ \\
Ortostase & 1,00 & - & - \\
Marcha & 0,99 & $(0,98-0,99)$ & $<0,001$ \\
Pontuação Total & 0,99 & $(0,98-0,99)$ & $<0,001$ \\
\hline
\end{tabular}

${ }^{*}$ CCl: Coeficiente de Correlação Intraclasse

\section{Discussão}

O presente estudo ampliou a avaliação das propriedades psicométricas da EMH, encontrando uma forte associação com a ERm e alto grau de confiabilidade quando aplicada através de entrevista. Este instrumento, desenvolvido para avaliar de forma específica a mobilidade, mostrou-se válido e confiável para avaliação de pacientes após AVC na fase aguda.

A validade concorrente da EMH foi demonstrada por uma forte correlação positiva com a ERm, revelando que quanto maior o comprometimento da mobilidade avaliada pela $\mathrm{EMH}$, maior o grau de incapacidade funcional. A ERm é um instrumento que avalia a incapacidade global sendo amplamente utilizado na prática clínica e em pesquisas ${ }^{910}$. Uma pesquisa apontou a ERm como a escala mais utilizada para avaliação de desfecho funcional em

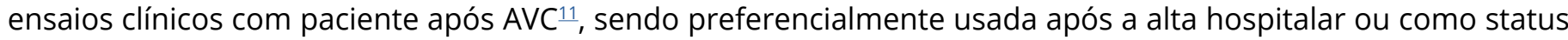
funcional antes do AVC? 
Sabe-se que o método de aquisição de dados é de grande importância, principalmente em pesquisa científicas, pois interfere diretamente nos custos e na logística da coleta de dados. As entrevistas, apesar de serem geralmente menos fidedignas, são mais rápidas, não requerem instrumentos e facilitam a logística da pesquisa, podendo em alguns casos ser aplicada por telefone ${ }^{12}$. O presente estudo mostrou que os escores da EMH quando aplicados por entrevista são muito semelhantes aos encontrados com a observação do desempenho, provalvelmente por que os seus itens são simples e objetivos. A avaliação da mobilidade através de entrevista não restringiria a coleta de dados aos profissionais que tem habilidade e autorização para retirar os pacientes do leito, poderia ser aplicada por outros profissionais de saúde, estudantes ou pesquisadores que não fazem parte da equipe assistencial da unidade onde o paciente encontra-se internado. Um exemplo encontrado na pratica clínica e na pesquisa é o Índice de Barthel, que é amplamente utilizado e pode ser aplicado através de entrevista com o paciente ou com um informante, não sendo imprescindível observar o paciente executando as tarefas $\frac{13}{3}$. Não foram encontrados registros que refiram a possibilidade das demais escalas de mobilidade validadas para pacientes após AVC, poderem ser aplicadas através de entrevistas 23,14,15.

Dentre as outras escalas de mobilidade validadas para população após AVC destacam-se a Mobility Scale for Acute Stroke e a Elderly Mobility Scale ${ }^{2,3}$. Esses instrumentos tiveram sua validade confiabilidade e validade de construto comprovada em diferentes estudos $^{2,3,16}$. Apesar de serem escalas simples e com tarefas semelhantes à EMH, a aplicação da Mobility Scale for Acute Stroke exige ambiente padronizado, como uma cadeira com $43 \mathrm{~cm}$ de altura, cama ajustável e apoio para os pés, o que poderia dificultar a inclusão desta escala na rotina diária de atendimentos ${ }^{2}$. A Elderly Mobility Scale inclui dentre as suas tarefas o teste de alcance funcional 3 , o que torna sua aplicação mais complexa, especialmente em pacientes com dificuldade de compreensão. Por outro lado, a Functional Status Score for the ICU, desenvolvida para pacientes internados em unidade de terapia intensiva, não necessita de ambiente padronizado ou equipamentos ${ }^{17}$, mas nesta escala a classificação de cada nível é mais detalhada do que na EMH, necessitando de maior tempo para sua aplicação.
Para este estudo, optamos pela comparação da EMH com a ERm, mesmo reconhecendo as limitações do uso da ERm na fase aguda, relacionadas a dificuldade em avaliar as atividades de vida diária e atividades habituais no ambiente hospitalar. No momento do desenvolvimento da $\mathrm{EMH}$, não foram identificadas outras escalas de mobilidade traduzidas e validadas para população brasileira para essa comparação. Estudos futuros comparando a EMH com outras escalas de mobilidade poderiam contribuir para avaliação das suas propriedades psicométricas.

\section{Conclusão}

A Escala de Mobilidade Hospitalar, projetada especificamente para pacientes após AVC, mostrou um alto grau de validade concorrente e se mostrou confiável quando aplicada através de entrevista.

\section{Agradecimentos}

À Coordenação de Fisioterapia do Hospital Geral Roberto Santos pelo apoio ao desenvolvimento dessa pesquisa e a todos os fisioterapeutas e estudantes que deram suporte durante a coleta de dados, sendo estes: Carla Suelen de Andrade, Caroline de Souza, Catiusque Brito, Manoela Rios, Lemuel Bernardes e Silvania Santos.

\section{Contribuições dos autores}

Maso I participou da concepção e delineamento do estudo, gerenciamento do banco de dados, da análise estatística dos dados e redação do artigo. Oliveira-Filho J participou na concepção e delineamento do estudo e da análise estatística dos dados. Jesus APJ participou no delineamento do estudo, definições de casos de AVC e interpretação dos dados. Monteiro M participou no delineamento do estudo e interpretação dos dados. Mascarenhas L, Makhoul M e Vasconcelos L participaram na aquisição e interpretação dos dados. Pinto EB participou da concepção e delineamento do estudo, coordenação do estudo e redação do artigo. Todos os autores aprovaram a versão final manuscrito.

\section{Conflitos de interesses}

Nenhum conflito financeiro, legal ou político envolvendo terceiros (governo, empresas e fundações privadas, etc.) foi declarado para nenhum aspecto do trabalho submetido (incluindo, mas não se limitando a subvenções e financiamentos, participação em conselho consultivo, desenho de estudo, preparação de manuscrito, análise estatística, etc.). 


\section{Referências}

1. Maso I, Pinto EB, Monteiro M, Makhoul M, Mendel T, Jesus PAP et al. A Simple Hospital Mobility Scale for Acute Ischemic Stroke Patients Predicts Long-term Functional Outcome. Neurorehabil Neural Repair. 2019;33(8):614-622. doi: $\underline{10.1177 / 1545968319856894}$

2. Simondson JA, Goldie P, Brock K, Nosworthy J. The Mobility Scale for Acute Stroke Patients: intra-rater and inter-rater reliability. Clin Rehabil. 1996;10:295-300. doi:

\subsection{7/026921559601000406}

3. Smith R. Validation and reliability of the Elderly Mobility Scale. Physiotherapy. 1994;80(11):744-747. doi: 10.1016/S00319406(10)60612-8

4. Hodgson C, Needham D, Haines K, Bailey M, Ward A, Harrold M et al. Feasibility and inter-rater reliability of the ICU Mobility Scale [published correction appears in Heart Lung. 2014;43(4):388]. Heart Lung. 2014;43(1):19-24. doi: 10.1016/j.hrtIng.2013.11.003

5. Hoyer EH, Friedman M, Lavezza A, Wagner-Kosmakos K, Lewis-Cherry R, Skolnik JL et al. Promoting mobility and reducing length of stay in hospitalized general medicine patients: A qualityimprovement project. J Hosp Med. 2016;11(5):341-7. doi: $10.1002 /$ jhm.2546

6. Cincura C, Pontes-Neto OM, Neville IS, Mendes HF, Menezes DF, Mariano DC et al. Validation of the National Institutes of Health Stroke Scale, modified Rankin Scale and Barthel Index in Brazil: the role of cultural adaptation and structured interviewing. Cerebrovasc Dis. 2009;27(2):119-22. doi: 10.1159/000177918

7. Callegari-Jacques SM. Bioestatística: Princípios e aplicações. Porto alegre: Editora Artmed; 2009.

8. Pilatti LA, Pedroso B, Gutierrez GL. Propriedades Psicométricas de Instrumentos de Avaliação: Um debate necessário. Revista Brasileira de Ensino de Ciência e Tecnologia. 2010;3(1):81-91. doi: 10.3895/S1982-873X2010000100005
9. Harrison JK, McArthur KS, Quinn TJ. Assessment scales in stroke: clinimetric and clinical considerations. Clin Interv Aging. 2013;8:201-11. doi: 10.2147/CIA.S32405

10. Banks JL, Marotta CA. Outcomes validity and reliability of the modified Rankin scale: implications for stroke clinical trials: a literature review and synthesis. Stroke. 2007;38(3):1091-6. doi: 10.1161/01.STR.0000258355.23810.c6

11. Quinn TJ, Dawson J, Walters MR, Lees KR. Functional outcome measures in contemporary stroke trials. Int J Stroke. 2009;4(3):200-5. doi: 10.1111/j.1747-4949.2009.00271.x

12. Barak S, Duncan PW. Issues in selecting outcome measures to assess functional recovery after stroke. NeuroRx. 2006;3(4):50524. doi: $10.1016 /$ j.nurx.2006.07.009

13. Quinn TJ, Langhorne P, Stott DJ. Barthel index for stroke trials: development, properties, and application. Stroke. 2011;42(4):1146-51. doi: 10.1161/STROKEAHA.110.598540

14. Linder A, Winkvist L, Nilsson L, Sernert N. Evaluation of the Swedish version of the Modified Elderly Mobility Scale (Swe M-EMS) in patients with acute stroke. Clin Rehabil. 2006;20(7):58497. doi: $10.1191 / 0269215506 \mathrm{cr} 9720 \mathrm{a}$

15. Tinl ML, Kale MK, Doshi S, Guarino AJ, Beninato M. The Mobility Scale for Acute Stroke predicts discharge destination after acute hospitalization. J Rehabil Med. 2014;46(3):219-24. doi: 10.2340/16501977-1269

16. Simondson JA, Goldie P, Greenwood KM. The Mobility Scale for Acute Stroke Patients: concurrent validity. Clin Rehabil. 2003;17(5):558-64. doi: 10.1191/0269215503cr650oa

17. Silva VZM, Araújo Neto JA, Cipriano Jr G, Pinedo M, Needham DM, Zanni JM et al. Brazilian version of the Functional Status Score for the ICU: translation and cross-cultural adaptation. Versão brasileira da Escala de Estado Funcional em UTI: tradução e adaptação transcultural. Rev Bras Ter Intensiva. 2017;29(1):34-38. doi: $10.5935 / 0103-507 X .20170006$ 